\title{
TRICHLOROTRIAZINE AS A SIMPLE AND EFFICIENT CATALYST PROMOTER FOR THE SYNTHESIS OF NEW GENERATION OF COUMARINS
}

\author{
MOHAMMAD NIKPASSAND ${ }^{*}$, , LEILA ZARE FEKRI ${ }^{b}$, NARJES CHANGIZ $^{a}$, FOROUZAN IMANI ${ }^{a}$ \\ ${ }^{a}$ Department of Chemistry, Rasht Branch, Islamic Azad University, Rasht, Iran \\ ${ }^{b}$ Department of Chemistry, Payame Noor University, PO Box 19395-3697 Tehran, Iran
}

(Received: June 20, 2013 - Accepted: July 8, 2013)

\begin{abstract}
The first synthesis of azo bearing coumarins from azo aldehydes and malononitrile in the presence of an efficient catalyst, trichlorotriazine under ultrasound irradiation in high yield and short reaction time is reported. In the other study, the first electrophilic substitution under ultrasound irradiation for the synthesis of azo bearing coumarins was developed. All of synthesized compounds were characterized by FTIR, NMR and elemental analysis.
\end{abstract}

Keywords: Coumarins, trichlorotriazine, malononitrile, azo aldehydes

\section{INTRODUCTION}

Coumarins are well known natural products for their diverse biological activity such as photosensitizes ${ }^{1}$, anti-HIV agents ${ }^{2}$, antibiotics ${ }^{3}$, rodenticides and oral anticoagulants ${ }^{4}$, inhibitor of platelet aggregation ${ }^{5}$, antibacterial ${ }^{6}$, anticancer ${ }^{7}$ and inhibitor of steroid $5 \alpha$ - reductase ${ }^{8}$. Coumarin derivatives display a broad range of applications to the pharmaceutical, perfume, cosmetic, food, agrochemical ${ }^{9}$, sensors and sophisticated photophysical system ${ }^{10-12}$, that is, in organic light-emitting diodes ${ }^{13}$ and molecular photonic devices ${ }^{14}$ and in the preparation of insecticides, optical brightening agents, dispersed fluorsent and tunable dye lasers ${ }^{15}$. Coumarins also act as intermediates for the synthesis of fluorocoumarins, chromenes, coumarones and 2-acylresorcinols ${ }^{16}$.

1 Coumarins have been synthesized by several routes including Pechman ${ }^{17}$, Knovenagel ${ }^{18}$, Reformatsky ${ }^{19}$ and Wittig reaction ${ }^{20}$.

On the other hand, various procedures were used for the Perkin synthesis of coumarins. The main disadvantages of the processes using these catalysts are long reaction times, low yields, harsh reaction condition and temperature above $150^{\circ} \mathrm{C}$, non reusability of the catalyst, use of excess amount of the reagent, strictly reactive condition( $\mathrm{N}_{2}$ atmosphere), special efforts required to prepare the catalyst and tedious work up and low selectivity.

11 However, to the best of our knowledge, no reports concerning coumarins conjugated with azo systems have appeared so far. Hence, synthesis of new generation of coumarins in fast and environmentally benign synthetic routes has created a lot of interest in organic chemistry.

\section{EXPERIMENTAL}

\section{Materials and measurements}

Melting points were measured on an Electrothermal 9100 apparatus. IR spectra were determined on a Shimadzo IR-470 spectrometer. ${ }^{1} \mathrm{H}$ NMR spectra were recorded on a $500 \mathrm{MHz}$ Bruker DRX-500 and ${ }^{13} \mathrm{C}$ NMR spectra were recorded on a $250 \mathrm{MHz}$ Bruker DRX-500 in DMSO-d as solvent. Chemicals were purchased from Merck and Fluka. Elemental analyses were done on a Carlo-Erba EA1110CNNO-S analyser and agreed with the calculated values. All solvents used were dried and distilled according to standard procedures.

General Procedure for the synthesis of azo conjugated coumarins 3 in pathway a under ultrasound irradiation

A mixture of azo aldehyde $1(1 \mathrm{mmol})$, malononitrile $(1 \mathrm{mmol})$ and TCT $(0.3 \mathrm{mmol})$ in $5 \mathrm{~mL} \mathrm{H}_{2} \mathrm{O}$ were placed into Pyrex-glass open vessel and irradiated in a water bath under silent condition by ultrasound $(45 \mathrm{kHz})$ at $60^{\circ} \mathrm{C}$ for the required reaction times $(5-9 \mathrm{~min})$. The progress of the reaction was monitored by TLC (EtOAc: petroleum ether 1:2). After completion of the reaction, as indicated by TLC, the reaction mixture was filtered to separate the catalyst and the organic compound was recrystallized by EtOH to produce azo coumarin derivatives $\mathbf{3 a - j}$ as pure crystalline products.

General Procedure for the synthesis of azo conjugated coumarins 3 in pathway $b$ under ultrasound irradiation

A mixture of various aniline $4(1 \mathrm{mmol}), \mathrm{NaNO}_{2}(1 \mathrm{mmol})$ and $\mathrm{HCl}(1 \mathrm{~mL})$ were placed into Pyrex-glass open vessel and irradiated in a water bath under silent condition by ultrasound $(45 \mathrm{kHz})$ at room temperature for the required reaction times $(5 \mathrm{~min})$. The progress of the reaction was monitored by TLC (EtOAc: petroleum ether 1:2). Then the reaction mixture was added in situ dropwise to synthesized compound 7, and the reaction was irradiated for the required reaction time ( $7-15 \mathrm{~min}$ ) to produce compound $\mathbf{3 a} \mathbf{a} \mathbf{j}$. Then the reaction mixture was cooled to room temperature, and the product was filtered without further purification.

2-oxo-6-(phenyldiazenyl)-2H-chromene-4-carbonitrile $3 a$ (table 2, entry 1) brown solid, $\mathrm{mp} 263^{\circ} \mathrm{C}$ dec. FT- IR (KBr): v 3055, $2231(\mathrm{CN})$, $1733(\mathrm{C}=\mathrm{O}), 1614,1560,1475,1180 \mathrm{Cm}^{-1} .{ }^{1} \mathrm{H} \mathrm{NMR}\left(\mathrm{CDCl}_{3}, 400 \mathrm{MHz}\right) \mathrm{d}_{\mathrm{H}}=$ 7.57-7.62 (m, 4H), 7.97-7.99 (m, 2H), 8.19 (d, $J=2.2 \mathrm{~Hz}, 1 \mathrm{H}), 8.33(\mathrm{dd}, J=$ $8.8 \mathrm{~Hz}, J=2.2 \mathrm{~Hz}, 1 \mathrm{H}), 8.41(\mathrm{~s}, 1 \mathrm{H}) \mathrm{ppm} .{ }^{13} \mathrm{C}$ NMR (DMSO- d, $100 \mathrm{MHz}$ ) $\mathrm{d}_{\mathrm{C}}=103.85,114.90,118.77,122.51,124.93,128.94,130.25,137.11,148.80$, $149.09,150.71,153.65,156.18,167.98$ ppm. Anal. Calcd. for $\mathrm{C}_{17} \mathrm{H}_{11} \mathrm{~N}_{3} \mathrm{O}_{2}: \mathrm{C}$, 70.58; H, 3.83; N, 14.53. Found: C, 70.44; H, 3.27; N, 14.45 .

2-oxo-6-(p-tolyldiazenyl)-2H-chromene-4-carbonitrile 3b (table 2, entry 2) brown solid, $\mathrm{mp} 263^{\circ} \mathrm{C}$ dec. FT- IR (KBr:) v 3049, 2225 (CN), 1737 $(\mathrm{C}=\mathrm{O}), 1608,1469,1412,1103 \mathrm{Cm}^{-1} .{ }^{1} \mathrm{H}$ NMR $\left(\mathrm{CDCl}_{3}, 400 \mathrm{MHz}\right) \mathrm{d}_{\mathrm{H}}=2.50(\mathrm{~s}$, $3 \mathrm{H}), 7.29(\mathrm{~d}, J=8.0 \mathrm{~Hz}, 2 \mathrm{H}), 7.55(\mathrm{~d}, J=8.8 \mathrm{~Hz}, 1 \mathrm{H}), 7.87(2 \mathrm{H}, \mathrm{d}, J=8.0 \mathrm{~Hz})$, $8.16(1 \mathrm{H}, \mathrm{d}, J=2.2 \mathrm{~Hz}), 8.31(1 \mathrm{H}, \mathrm{dd}, J=8.8 \mathrm{~Hz}, J=2.2 \mathrm{~Hz}), 8.39(\mathrm{~s}, 1 \mathrm{H}) \mathrm{ppm}$. ${ }^{13} \mathrm{C}$ NMR (DMSO- $\left.\mathrm{d}_{6}, 100 \mathrm{MHz}\right) \mathrm{d}_{\mathrm{c}}=20.12,105.63,115.78,119.09,123.11$, 124.73, 129.64, 130.16, 139.45, 148.34,149.87, 150.11, 152.45, 156.90, 166.08 ppm. Anal. Calcd. for $\mathrm{C}_{17} \mathrm{H}_{11} \mathrm{~N}_{3} \mathrm{O}_{2}: \mathrm{C}, 70.58 ; \mathrm{H}, 3.83 ; \mathrm{N}, 14.53$. Found: C, 70.44; H, 3.27; N, 14.45 .

6-((4-methoxyphenyl)diazenyl)-2-oxo-2H-chromene-4-carbonitrile 3c (table 2, entry 3) brown solid, mp $263^{\circ} \mathrm{C} \mathrm{dec} .{ }^{1} \mathrm{H}$ NMR $\left(\mathrm{CDCl}_{3}, 400 \mathrm{MHz}\right) \mathrm{d}_{\mathrm{H}}=$ $3.96(\mathrm{~s}, 3 \mathrm{H}), 7.08(\mathrm{~d}, J=8.8 \mathrm{~Hz}, 2 \mathrm{H}), 7.53(\mathrm{~d}, J=8.8 \mathrm{~Hz}, 1 \mathrm{H}), 7.98(\mathrm{~d}, J=8.8$ $\mathrm{Hz}, 2 \mathrm{H}), 8.12(\mathrm{~d}, J=2.0 \mathrm{~Hz}, 1 \mathrm{H}), 8.28(\mathrm{dd}, J=8.8 \mathrm{~Hz}, J=2.0 \mathrm{~Hz}, 1 \mathrm{H}), 8.39$ $(\mathrm{s}, 1 \mathrm{H}) \mathrm{ppm} .{ }^{13} \mathrm{C}$ NMR (DMSO- $\left.\mathrm{d}_{6}, 100 \mathrm{MHz}\right) \mathrm{d}_{\mathrm{C}}=71.00,106.33,118.91$, $119.09,124.01,126.82,128.93,130.65,138.53,149.24,149.87,151.13$, 154.46, 156.89, 167.61 ppm. Anal. Calcd. for $\mathrm{C}_{17} \mathrm{H}_{11} \mathrm{~N}_{3} \mathrm{O}_{3}: \mathrm{C}, 70.58 ; \mathrm{H}, 3.83 ; \mathrm{N}$, 14.53. Found: C, 70.36; H, 3.78; N, 14.39 .

6-((4-chlorophenyl)diazenyl)-2-oxo-2H-chromene-4-carbonitrile 3d (table 2, entry 4) brown solid, $\mathrm{mp} 300^{\circ} \mathrm{C}$ dec. FT- IR $(\mathrm{KBr}) v: 2933,2233$ $(\mathrm{CN}), 1737(\mathrm{C}=\mathrm{O}), 1610,1650,1510,1085 \mathrm{Cm}^{-1} .{ }^{1} \mathrm{H} \mathrm{NMR}\left(\mathrm{CDCl}_{3}, 400 \mathrm{MHz}\right)$ $\mathrm{d}_{\mathrm{H}}=7.55(\mathrm{~d}, J=8.6 \mathrm{~Hz}, 2 \mathrm{H}), 7.59(\mathrm{~d}, J=8.4 \mathrm{~Hz}, 1 \mathrm{H}), 7.93(\mathrm{~d}, J=8.6 \mathrm{~Hz}, 2 \mathrm{H})$, $8.19(\mathrm{~d}, J=2.4 \mathrm{~Hz}, 1 \mathrm{H}), 8.32(\mathrm{dd}, J=8.6 \mathrm{~Hz}, J=2.4 \mathrm{~Hz}, 1 \mathrm{H}), 8.42(\mathrm{~s}, 1 \mathrm{H}) \mathrm{ppm}$. ${ }^{13} \mathrm{C}$ NMR (DMSO- $\mathrm{d}_{6}, 100 \mathrm{MHz}$ ) $\mathrm{d}_{\mathrm{C}}=103.85,114.90,118.77,122.51,124.93$, $128.94,130.25,137.11,148.80,150.71,153.65,156.18,156.98,167.65 \mathrm{ppm}$. Anal. Calcd. for $\mathrm{C}_{16} \mathrm{H}_{8} \mathrm{ClN}_{3} \mathrm{O}_{2}: \mathrm{C}, 62.05 ; \mathrm{H}, 2.60 ; \mathrm{N}, 13.57$. Found: $\mathrm{C}, 62.98$; $\mathrm{H}, 2.57 ; \mathrm{N}, 13.53$.

6-((4-bromophenyl)diazenyl)-2-oxo-2H-chromene-4-carbonitrile $3 \mathrm{e}$ (table 2, entry 5) brown solid, $\mathrm{mp} 275^{\circ} \mathrm{C}$ dec. FT- IR (KBr) v: 2929, $2237(\mathrm{CN})$, $1739(\mathrm{C}=\mathrm{O}), 1685,1652,1614,1506 \mathrm{Cm}^{-1} .{ }^{1} \mathrm{H}$ NMR $\left(\mathrm{CDCl}_{3}, 400 \mathrm{MHz}\right) \mathrm{d}_{\mathrm{H}}=$ $7.57(\mathrm{~d}, J=8.8 \mathrm{~Hz}, 1 \mathrm{H}), 7.72(\mathrm{~d}, J=8.8 \mathrm{~Hz}, 2 \mathrm{H}), 7.88(\mathrm{~d}, J=8.8 \mathrm{~Hz}, 2 \mathrm{H}), 8.19$ $(\mathrm{d}, J=2.0 \mathrm{~Hz}, 1 \mathrm{H}), 8.33(\mathrm{dd}, J=8.8 \mathrm{~Hz}, J=2.0 \mathrm{~Hz}, 1 \mathrm{H}), 8.42(\mathrm{~s}, 1 \mathrm{H}) \mathrm{ppm} \cdot{ }^{13} \mathrm{C}$ NMR (DMSO- $\left.\mathrm{d}_{6}, 100 \mathrm{MHz}\right) \mathrm{d}_{\mathrm{C}}=103.81,114.88,118.77,124.98,126.11$, $128.91,132.56,132.98,148.79,150.98,153.64,156.16,156.97,167.78 \mathrm{ppm}$. Anal. Calcd. For $\mathrm{C}_{16} \mathrm{H}_{8} \mathrm{BrN}_{3} \mathrm{O}_{2}$ : C, 54.26; H, 2.28; N, 11.86. Found: C, 54.68; $\mathrm{H}, 2.56 ; \mathrm{N}, 11.09$. 
6-((4-nitrophenyl)diazenyl)-2-oxo-2H-chromene-4-carbonitrile $3 \mathrm{f}$ (table 2, entry 6) brown solid, $\mathrm{mp} 274^{\circ} \mathrm{C}$ dec. FT- IR (KBr) v: 3047, 2921 , $2231(\mathrm{CN}), 1735(\mathrm{C}=\mathrm{O}), 1610,1562,1463,1182 \mathrm{Cm}^{-1}$. ${ }^{1} \mathrm{H} \mathrm{NMR}\left(\mathrm{CDCl}_{3}, 400\right.$ MHz) $\mathrm{d}_{\mathrm{H}}=7.57(\mathrm{~d}, J=8.8 \mathrm{~Hz}, 1 \mathrm{H}), 7.71(\mathrm{~d}, J=8.6 \mathrm{~Hz}, 2 \mathrm{H}), 7.94(\mathrm{~d}, J=8.4 \mathrm{~Hz}$, $2 \mathrm{H}), 8.19(\mathrm{~d}, J=2.0 \mathrm{~Hz}, 1 \mathrm{H}), 8.32(\mathrm{dd}, J=8.8 \mathrm{~Hz}, J=2.2 \mathrm{~Hz}), 8.40(\mathrm{~s}, 1 \mathrm{H})$ ppm. ${ }^{13} \mathrm{C}$ NMR (DMSO- $\mathrm{d}_{6}, 100 \mathrm{MHz}$ ) $\mathrm{d}_{\mathrm{C}}=107.23,116.76,118.56,126.99$, $127.09,128.34,132.06,132.56,148.45,152.14,153.67,156.56,156.97$ 167.87 ppm. Anal. Calcd. For $\mathrm{C}_{16} \mathrm{H}_{8} \mathrm{~N}_{4} \mathrm{O}_{4}: \mathrm{C}, 60.00 ; \mathrm{H}, 2.52 ; \mathrm{N}, 17.49$. Found: C, $60.23 ; \mathrm{H}, 2.59 ; \mathrm{N}, 17.88$

6-((2-methyl-4-nitrophenyl)diazenyl)-2-oxo-2H-chromene-4carbonitrile $3 \mathrm{~g}$ (table 2 , entry 7) brown solid, $\mathrm{mp} 265^{\circ} \mathrm{C}$ dec. FT- IR $(\mathrm{KBr})$ $v: 3053,2229(\mathrm{CN}), 1733(\mathrm{C}=\mathrm{O}), 1610,1519,1429,1178 \mathrm{Cm}^{-1}$. ${ }^{1} \mathrm{H}$ NMR $\left(\mathrm{CDCl}_{3}, 400 \mathrm{MHz}\right) \mathrm{d}_{\mathrm{H}}=2.86(\mathrm{~s}, 3 \mathrm{H}), 7.61(\mathrm{~d}, J=8.8 \mathrm{~Hz}, 1 \mathrm{H}), 7.78(\mathrm{~d}, J=8.8$ $\mathrm{Hz}, 1 \mathrm{H}), 8.18(\mathrm{dd}, J=8.8 \mathrm{~Hz}, J=2.0 \mathrm{~Hz}, 1 \mathrm{H}), 8.25(\mathrm{~d}, J=2.0 \mathrm{~Hz}, 1 \mathrm{H}), 8.30(\mathrm{~d}$ $J=2.0 \mathrm{~Hz}, 1 \mathrm{H}), 8.35(\mathrm{dd}, J=8.8 \mathrm{~Hz}, J=2.0 \mathrm{~Hz}), 8.44(\mathrm{~s}, 1 \mathrm{H}) \mathrm{ppm} .{ }^{13} \mathrm{C} \mathrm{NMR}$ (DMSO- $\left.\mathrm{d}_{6}, 100 \mathrm{MHz}\right) \mathrm{d}_{\mathrm{C}}=15.52,103.86,114.84,117.34,118.87,122.69$, $126.90,128.89,132.08,137.53,139.76,149.00,149.19,153.51,156.60$, $156.92,162.88$ ppm. Anal. Calcd. For $\mathrm{C}_{17} \mathrm{H}_{10} \mathrm{~N}_{4} \mathrm{O}_{4}: \mathrm{C}, 61.08 ; \mathrm{H}, 3.02 ; \mathrm{N}, 16.76$. Found: C, 61.35; H, 3.46; N, 16.90 .

6-((2-nitrophenyl)diazenyl)-2-oxo-2H-chromene-4-carbonitrile $3 \mathrm{~h}$ (table 2, entry 8) brown solid, mp $265^{\circ} \mathrm{C}$ dec. FT-IR (KBr) v: 3040,2233 , $1731,1608,1566,1461,1178 \mathrm{Cm}^{-1} .{ }^{1} \mathrm{H}$ NMR $\left(\mathrm{CDCl}_{3}, 400 \mathrm{MHz}\right) \mathrm{d}_{\mathrm{H}}=7.42$ $(\mathrm{t}, J=8.0 \mathrm{~Hz}, 1 \mathrm{H}), 7.50(\mathrm{t}, J=6.8 \mathrm{~Hz}, 1 \mathrm{H}), 7.59(\mathrm{~d}, J=8.9 \mathrm{~Hz}), 7.64(\mathrm{~d}, J$ $=7.6 \mathrm{~Hz}, 1 \mathrm{H}), 7.77(\mathrm{~d}, J=2.0 \mathrm{~Hz}, 1 \mathrm{H}), 8.23(\mathrm{~d}, J=1.8 \mathrm{~Hz}, 1 \mathrm{H}), 8.38(\mathrm{dd}$, $J=8.9, \quad J=1.8 \mathrm{~Hz}), 8.44(\mathrm{~s}, 1 \mathrm{H}) \mathrm{ppm} .{ }^{13} \mathrm{C}$ NMR (DMSO- $\left.\mathrm{d}_{6}, 100 \mathrm{MHz}\right)$ $\mathrm{d}_{\mathrm{C}}=103.84,114.76,118.12,118.68,118.87,125.56,128.79,131.38,133.82$, $134.79,142.08,149.04153 .68,156.36,156.94,165.98 \mathrm{ppm}$. Anal. Calcd. For
$\mathrm{C}_{16} \mathrm{H}_{8} \mathrm{~N}_{4} \mathrm{O}_{4}: \mathrm{C}, 60.00 ; \mathrm{H}, 2.52 ; \mathrm{N}, 17.49$; Found: C, 60.57; H, 2.43; N, 17.05. 6-((2-chlorophenyl)diazenyl)-2-oxo-2H-chromene-4-carbonitrile $3 \mathrm{i}$ (table 2, entry 9) brown solid, $\mathrm{mp} 275^{\circ} \mathrm{C}$ dec. FT-IR (KBr) v: 3043, 2231 $(\mathrm{C} \equiv \mathrm{N}), 1733(\mathrm{C}=\mathrm{O}), 1610,1566,1463,1178 \mathrm{~cm}^{-1} .{ }^{1} \mathrm{H} \mathrm{NMR}\left(\mathrm{CDCl}_{3}, 400\right.$ MHz) $\mathrm{d}_{\mathrm{H}}=7.41(\mathrm{t}, J=7.2 \mathrm{~Hz}, 1 \mathrm{H}), 7.50(\mathrm{t}, J=7.6 \mathrm{~Hz}, 1 \mathrm{H}), 7.58(\mathrm{~d}, J=8.8 \mathrm{~Hz}$, $1 \mathrm{H}), 7.63(\mathrm{~d}, J=8.0 \mathrm{~Hz}, 1 \mathrm{H}), 7.77(\mathrm{~d}, J=8.0 \mathrm{~Hz}, 1 \mathrm{H}), 8.23(\mathrm{~d}, J=1.6 \mathrm{~Hz}, 1 \mathrm{H})$, 8.37 (dd, $J=8.8,1.6 \mathrm{~Hz}), 8.44(\mathrm{~s}, 1 \mathrm{H}) \mathrm{ppm} .{ }^{13} \mathrm{C}$ NMR (DMSO- d, $\left.100 \mathrm{MHz}\right)$ $\mathrm{d}_{\mathrm{C}}: 106.56,115.87,117.81,118.08,118.23,125.67,129.07,130.98,133.22$, $137.45,142.63,148.46,154.31,156.63,156.94,165.75 \mathrm{ppm}$. Anal. Calcd. For $\mathrm{C}_{16} \mathrm{H}_{8} \mathrm{ClN}_{3} \mathrm{O}_{2}$ : C, 62.05; H, 2.60; N, 13.57; Found: C, 62.45; H, 2.24; N, 13.41. 6-((4-iodophenyl)diazenyl)-2-ox 0-2H-chromene-4-carbonitrile 3j (table 2, entry 10) brown solid, mp $288^{\circ} \mathrm{C}$ dec. FT-IR (KBr) v: 3080 , $2231(\mathrm{C} \equiv \mathrm{N}), 1735(\mathrm{C}=\mathrm{O}), 1610,1560,1461,1182 \mathrm{~cm}^{-1} .{ }^{1} \mathrm{H}$ NMR $(400 \mathrm{MHz}$, $\left.\mathrm{CDCl}_{3}\right) \mathrm{d}_{\mathrm{H}}=7.57(\mathrm{~d}, J=8.8 \mathrm{~Hz}, 1 \mathrm{H}), 7.77(\mathrm{~d}, \mathrm{~J}=8.8 \mathrm{~Hz}, 2 \mathrm{H}), 7.94(\mathrm{~d}, J=8.8$ $\mathrm{Hz}, 2 \mathrm{H}), 8.19(\mathrm{~d}, J=2.2 \mathrm{~Hz}, 1 \mathrm{H}), 8.32(\mathrm{dd}, J=8.8,2.2 \mathrm{~Hz}, 1 \mathrm{H}), 8.40(\mathrm{~s}, 1 \mathrm{H})$ ppm. ${ }^{13} \mathrm{C}$ NMR (DMSO- $\left.\mathrm{d}_{6}, 100 \mathrm{MHz}\right) \mathrm{d}_{\mathrm{C}}=100.33,103.38,114.85,118.85$, $124.99,129.14,132.16,139.05,148.81,151.41,153.65,156.14,156.97$, 167.49 ppm. Anal. Calcd. For $\mathrm{C}_{16} \mathrm{H}_{8} \mathrm{IN}_{3} \mathrm{O}_{2}: \mathrm{C}, 47.90 ; \mathrm{H}, 2.01 ; \mathrm{N}, 10.47$; Found: C, $47.57 ; \mathrm{H}, 2.78 ; \mathrm{N}, 10.23$.

\section{RESULTS AND DISCUSSION}

In continuation of our studies on the synthesis of pharmaceutical and heterocyclic compound ${ }^{21-23}$, herein, we wish to report our preliminary efforts for the synthesis of new azo containing coumarin moiety from synthesized aldehydes and malononitrile using TCT under (i) reflux condition and (ii) ultrasound irradiation and in electrophilic substitution reaction (Scheme 1).

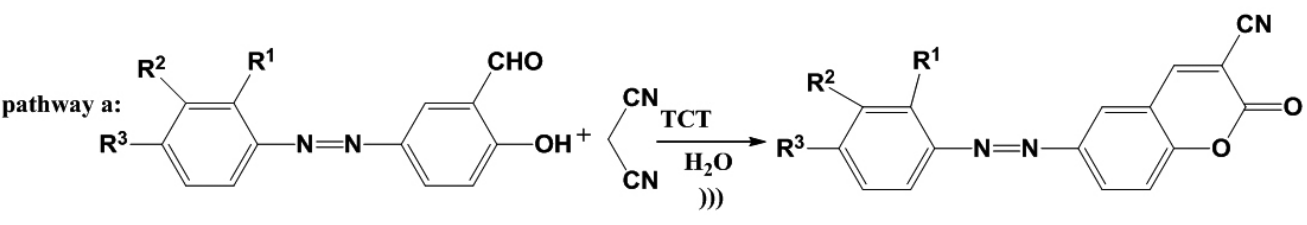

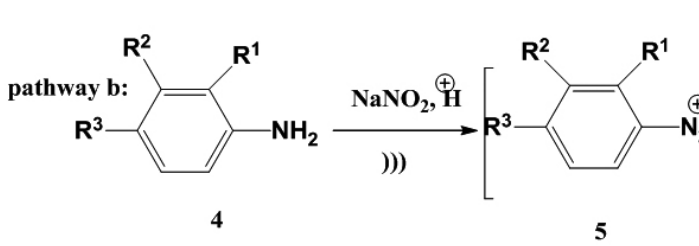

3a: $\mathbf{R}^{1}=\mathbf{R}^{2}=\mathbf{R}^{3=} \mathbf{H}$ 3b: $\mathbf{R}^{1}=\mathbf{R}^{2}=\mathbf{H}, \mathbf{R}^{3}=\mathrm{CH}_{3}$ 3c: $\mathbf{R}^{1}=\mathbf{R}^{2}=\mathrm{H}, \mathbf{R}^{3}=\mathrm{OCH}_{3}$ 3d: $\mathbf{R}^{1}=\mathbf{R}^{2}=\mathbf{H}, \mathbf{R}^{3}=\mathbf{C l}$ 3e: $\mathbf{R}^{1}=\mathbf{R}^{2}=\mathbf{H}, \mathbf{R}^{3}=\mathbf{B r}$

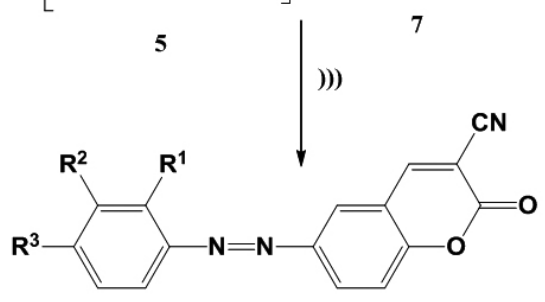

3f: $\mathbf{R}^{1}=\mathbf{R}^{2}=\mathrm{H}, \mathbf{R}^{3}=\mathrm{NO}_{2}$ 3g: $\mathbf{R}^{1}=\mathrm{CH}_{3}, \mathbf{R}^{2}=\mathrm{H}, \mathbf{R}^{3}=\mathrm{NO}_{2}$ 3h: $\mathbf{R}^{1}=\mathrm{NO}_{2}, \mathbf{R}^{2}=\mathbf{R}^{3}=\mathrm{H}$ 3i: $\mathbf{R}^{1}=\mathbf{C l}, \mathbf{R}^{2}=\mathbf{R}^{3}=\mathbf{H}$ $3 j: R^{1}=R^{2}=H, R^{3}=I$
Scheme1. Aqueous synthesis of coumarins using TCT.

Recently organic reactions in water without use of harmful organic solvent have drawn much more attention, because water is a cheap, safe and environmentally benign solvent. So, we prefer to use water as a green solvent in this reaction. Initially, we carried out the reaction of 2-hydroxy5 -(phenyldiazenyl)benzaldehyde (1a) and malononitrile (2) in the presence of various amount of TCT at reflux condition. The results are summarized in Table 1.
Table 1. The optimization of amount of catalyst for the synthesis of 3a at reflux.

\begin{tabular}{|c|c|c|c|c|}
\hline Entry & catalyst & $\begin{array}{c}\text { Amount of } \\
\text { catalyst }\end{array}$ & $\begin{array}{c}\text { Time } \\
(\mathrm{min})\end{array}$ & Yield(\%) \\
\hline 1 & none & none & 240 & 57 \\
\hline 2 & TCT & $0.1 \mathrm{mmol}$ & 75 & 67 \\
\hline 3 & TCT & $0.3 \mathrm{mmol}$ & 60 & 76 \\
\hline 4 & TCT & $0.5 \mathrm{mmol}$ & 60 & 74 \\
\hline
\end{tabular}

Based on the results above, the reaction condition was optimized to improve the yield and reaction time by changing the amount of catalyst. As shown in table 1 , when the reaction was performed in the absence of catalyst, $57 \%$ yield of product was observed in 4 hours (entry 1, Table 1). However, 
when $0.3 \mathrm{mmol}$ of TCT was used, the yield was improved significantly (entry 3 , table 1). The reaction took much longer reaction time to give $3 \mathbf{a}$ when $0.1 \mathrm{mmol}$ of TCT was involved as catalyst. Higher amount of catalyst did not further improve the yield of 3a (entry4, Table 1).

To investigate the scope of this synthesis, different synthesized aldehydes were reacted with malononitrile in the presence of $0.3 \mathrm{mmol}$ of TCT at reflux condition and the results were listed in Table 2. The reaction preceded smoothly giving $71-81 \%$ yield with various synthesized aldehydes bearing different substituent. Moreover, azo linked aldehydes containing electron withdrawing group provided better yields and times than those with electron donating groups.

Ultrasound irradiation has been increasingly used in organic synthesis in last three decades. Comparing with traditional methods, this method is more conveniently and easily controlled. In the course of our investigation to develop a green and effective procedure to reach higher yield, shorter reaction time and milder reaction condition, we concentrated to synthesize product $\mathbf{3 a - j}$ under ultrasound irradiation. The results were summarized in Table 2. The higher yield and less reaction time during ultrasonic irradiation in the presence of the catalyst can be attributed to implosive collapse of the cavitations period of the sound waves. When the formed bubbles burst, it results in high temperature and high pressure which facilitate the intermolecular reaction. This is more useful when the solid act as a catalyst.

The possible mechanism for the synthesis of $\mathbf{3 a}$ in the presence of TCT as a promoter is shown in Scheme 2. On the basis of this mechanism, TCT catalyzes the Pechman reaction by the activation of the aldehyde, making the carbonyl group susceptible to nucleophilic attack by malononitrile by conversion to $\mathrm{HCl}$ in situ. The intramolecular treatment of $\mathrm{OH}$ to nitrile group and hydrolysis of intermediate 7 lead to product $\mathbf{3 a}$.

Table 2. Perkin synthesis of azo conjugated comarins $\mathbf{3 a - j}$.

\begin{tabular}{|c|c|c|c|c|c|c|c|}
\hline & & \multicolumn{2}{|c|}{ reflux } & \multicolumn{2}{|c|}{ ultrasound } & \multicolumn{2}{|c|}{ ultrasound } \\
\hline & & Time (min) & Yield $(\%)^{\mathrm{a}}$ & Time (min) & Yield $(\%)^{\mathrm{a}}$ & Time (min) & Yield $(\%)^{\mathrm{ab}}$ \\
\hline 1 & $3 a$ & 60 & 76 & 7 & 89 & 12 & 87 \\
\hline 2 & $3 b$ & 75 & 73 & 9 & 92 & 15 & 90 \\
\hline 3 & $3 c$ & 75 & 79 & 8 & 90 & 10 & 90 \\
\hline 4 & 3d & 60 & 75 & 5 & 95 & 8 & 94 \\
\hline 5 & $3 e$ & 60 & 80 & 5 & 96 & 7 & 97 \\
\hline 6 & $3 f$ & 45 & 81 & 5 & 98 & 9 & 95 \\
\hline 7 & $3 g$ & 75 & 77 & 7 & 86 & 13 & 82 \\
\hline 8 & $3 \mathrm{~h}$ & 75 & 71 & 8 & 88 & 10 & 86 \\
\hline 9 & $3 \mathbf{i}$ & 75 & 75 & 7 & 87 & 10 & 88 \\
\hline 10 & $3 \mathbf{j}$ & 60 & 80 & 5 & 96 & 9 & 97 \\
\hline
\end{tabular}

${ }^{a}$ Isolated yield. ${ }^{b}$ Identified by spectroscopic analysis (IR, ${ }^{1} \mathrm{H}$ NMR, ${ }^{13} \mathrm{C}$ NMR and elemental analysis)

All of the products were fully characterized by spectroscopic methods (IR and ${ }^{1} \mathrm{H}$ NMR, ${ }^{13} \mathrm{C}$ NMR and elemental analysis). All of the products 3 exhibited a singlet in their ${ }^{1} \mathrm{H}$ NMR spectra at $\delta=8.39-8.40 \mathrm{ppm}$ for deshield $\mathrm{H}_{\beta}$ in coumarin ring and also a distinguishing peak at $\delta=167 \mathrm{ppm}$ for $\mathrm{C}=\mathrm{O}$ in their ${ }^{13} \mathrm{C}$ NMR spectra. In IR spectra revealed $\mathrm{C}-\mathrm{O}, \mathrm{N}=\mathrm{N}, \mathrm{CN}$ and $\mathrm{C}=\mathrm{O}$ peak at $1178-1182,1566,2225-2237$ and $1731-1739 \mathrm{~cm}^{-1}$.

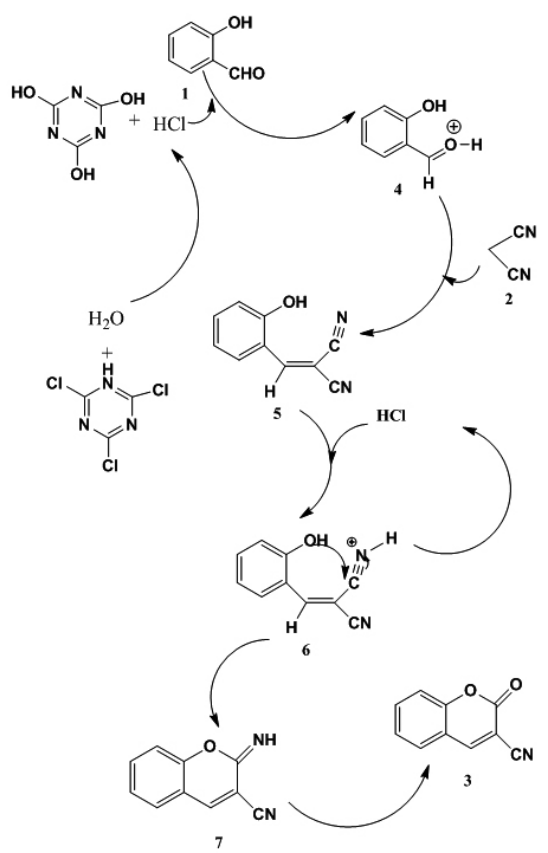

Scheme 2. Proposed pathway for the synthesis of azo containing coumarin.
In the final research, we were interested to synthesize compound $\mathbf{3}$ via electrophilic substitution. In this procedure, initially, the reaction between aldehyde and malononitrile was carried out, followed by drop-wise addition of diazonium salt to synthesized coumarins under ultrasound irradiation. Various derivatives of product 3 were synthesized in this pathway. The results are summarized in table 2. It was interesting that the reaction time and yield in this new pathway for the synthesis of azo containing coumarins were interesting as the former pathway. On the other hand, this reaction in this new pathway can be done at room temperature under ultrasound irradiation without any undesirable by product.

\section{ACKNOWLEDGEMENT}

We thank the Research Committee of Islamic Azad University of Rasht Branch for partial support given to this study.

\section{CONCLUSION}

TCT is an inexpensive, non corrosive and environmentally benign catalyst. Finally, a convenient and efficient procedure for the preparation of azo containing coumarins in high yield and short reaction time and mild condition under ultrasound irradiation was reported. The electrophilic substitution reaction reported in this work is mild and a wide range of functional groups can be tolerated in the synthesis coumarins. In this pathway, the purification process is much simplified. Generally, short reaction times and productivity, availability of reactants, operational simplicity and easy workup are the notable advantages of this methodology.

\section{REFERENCES}

1. Wulf, H. Rauer, H. During, T. Hanselmann, C. Ruff, K. Wrisch, A. Grissmer, S. Hansel, W. J. Med. Chem. 41, 4542, (1998).

2. Spino, C. Dodier, M. Sotheeswaren, S. Bioorg. Med. Chem. Lett. 8, 3475 , (1998).

3. Crow, F. W. Duholke, W. K. Farley, K. A. Hadden, C. E. Hahn D. A. 
Kaluzny, B. D. Mallory, C. S. Martin, G. E. Smith, R. F. Thamann, T. J. J. Heterocycl. Chem. 36, 365, (1999).

4. Barker, W. M. Hermodson, M. A. Link. K. P. J. Med. Chem.14, 167, (1971).

5. Cavettos, G. Nano, G. M. Palmisano, G. Tagliapietra, S. Tetrahedron: Asymmetry 12, 707, (2001).

6. Kayser, O. Kolodziej, H. Planta Med. 63, 508, (1997).

7. Wang, C. J. Hsieh, Y. J. Chu, C. Y. Lim, Y. L. Tseng, T. H. Cancer Lett. 183, 163, (2002).

8. Fan, G. J. Mar, W. Park, M. K. Choi, E. W. Kim, K. Kim, S. Bioorg. Med. Chem. Lett. 11, 2361, (2001)

9. Kennedy, R. O. Thornes, R. D. Coumarins: Biology, Applications and Mode of Action, John Wiley and Sons, Chichester, 1997.

10. Hara, K. Sayama, K. Ohga, Y. Shinpo, A. Suga, S. Arakawa, H. Chem. Commun. 569, (2001)

11. Hara, K. Tachibana, Y. Ohga, Y. Shinpo, A. Suga, S. Sayama, K.; Sugihara, H. Arakawa, H. Sol. Energy Mater. Sol. Cells 77, 89, (2003).
12. Jiao, C. Niu, C. Chen, L. Shen, G. Yu, R. Sens. Actuators B 94,176, ( 2003).

13. Mitsuya, M. Suzuki, T. Koyama, T. Appl. Phys. Lett. 77, 3272, (2000).

14. Adronov, A. Gilat, S. L. Frechet, J. M. J. Ohta, K. Neuwahl, F. V. R. Fleming, G. R. J. Am. Chem. Soc.122, 1175, ( 2000).

15. Maeda, M. Laser Dyes, Academic Press, New York, 1984.

16. Sethna, S. M. Shah, N. M. Chem. Rev. 36, 1, (1945).

17. Murray, R. D. Mendez, J. Brown, S. A. The Natural Coumarins: Occurrence, Chemistry and Biochemistry; John Wiley \& Sons: New York, 1982.

18. Johnson. J. R. Org. React. 1, 210, (1942).

19. Jones, G. Org. React. 15, 204, (1967).

20. Brafola, G; Fringuelli, F. Piermatti, O. Pizzo, F. Heterocycles 43, 1257, (1996).

21. Nikpassand, M. Mamaghani M. Tabatabaeian, K. Kupaei Abiazi, M. Mol. Diversity 389, (2009)

22. Nikpassand, M. Zare, L. Shafaati, T. Chin. J. Chem. 30, 604, (2012).

23. Nikpassand, M. Zare, L. Saberi, M. Monatsh. Chem. 143, 289, (2012). 